\title{
Le figement lexical au service du discours politique en Algérie. Les ॥ zones d'ombre ॥ entre euphémisation et structure lexicalisée
}

\author{
Set Phrases in the Service of Political Discourse in Algeria \\ The "Areas of Shadow" Between Euphemisation \\ and Lexicalized Structure
}

\author{
Maizi Sofiane \\ Université Blida 2 Lounici Ali, Algérie
}

\begin{abstract}
Résumé : Cet article propose d'analyser l'emploi de la redondance figée " الظل (les zones d'ombre) dans le discours politique algérien. II met en évidence la relation entretenue entre la tendance de lexicalisation de cette séquence et les stratégies politiques du discours d'autorité. Cette séquence, qui provient du discours politique, désigne euphémiquement des zones emblématiques de la misère et de l'exclusion sociale. Après la présentation du cadre théorique de l'analyse, cette étude s'intéresse à l'emploi de cette séquence figée dans le discours politique et médiatique algérien.
\end{abstract}

Mołs-clés : figement, euphémisme, lexicalisation, sens contextuel, néosémie.

\begin{abstract}
This article proposes to analyse the use of the fixed redundancy "مناطق الظل (areas of shadow) in the Algerian political discourse. It highlights the relationship maintained between the tendency of lexicalization of this sequence and the political strategies of the discourse of authority. This sequence, which comes from political discourse, designates euphemistically emblematic areas of misery and social exclusion. After presenting the theoretical framework of the analysis, this study focuses on the use of this frozen sequence in Algerian political and media discourse.
\end{abstract}

Keywords: figement, euphemism, lexicalization, contextual meaning, neosemia.

\section{Introduction}

Les discours politiques sont traversés par des redondances figées (Fiala, Habert, Lafon, \& Pineira-Tresmontant, 1987; Krieg-Planque, 2015; Tournier, 1985), indices du positionnement idéologiques des institutions qui les produisent, ou, plus exactement, qui les reproduisent. Ces redondances figées sont, essentiellement, l'objet de recherche de l'analyse du discours, mais de nombreuses ramifications des sciences du langage s'intéressent également à ces séquences répétées dans le discours 
politique, ce qui induit un éclatement terminologique (figement, lexicalisation, segments répétés, sloganisation, occurrence, collocation, néologie...). Dans cette contribution, nous retenons le terme de figement pour désigner ces modes d'expression spécifiques du discours politique. Cette notion sera le fil conducteur de notre réflexion dans cet article consacré à l'emploi de la séquence figée |"ناطق الطلاس dans le discours politique algérien.

Cette séquence figée, qui est un calque linguistique du français « zones d'ombres », est répétée inlassablement depuis une année dans le discours politique algérien. Elle a fait son irruption dans le discours politique le 16 février 2020 lors d'une réunion entre le président de la République algérienne, Abdelmajid Tebboune, et les 48 walis (préfets)'. Ce mot composé est le titre d'un documentaire de 35 minutes filmé par la télévision nationale algérienne sur commande de la présidence de la République. Depuis la projection de ce film, la formule rituelle est ressassée dans tous les journaux télévisés, les articles de presse, les communiqués officiels et les débats publics. Le succès médiatique de cette formule laconique interpelle le chercheur académique et pose des interrogations légitimes sur le sens contextuel de ce mot composé à partir du lexème arabe "الظل " " L'ombre dans la langue arabe a une connotation foncièrement appréciative ou positive, contrairement à la langue française où le mot composé «zones d'ombre » a souvent une connotation dépréciative. Ce néologisme de sens emprunté à la langue française, sans aucune considération des spécificités culturelles de la langue cible, serait-il une faute de traduction provoquée par une maîtrise approximative de la langue d'arrivée (arabe) ou bien s'agit-il d'un choix de traduction délibéré ? Pourquoi les auteurs du discours politique recourent à une néosémie pour exprimer une réalité qui peut être décrite aisément par des lexèmes de la langue arabe ? Le passage en force de cette séquence figée dans la langue cible (arabe standard en Algérie) et la persistance de son emploi dans le discours politique algérien sont-ils des indices de l'existence d'une stratégie politique visant à manipuler le public-cible?

\section{Mise au point méthodologique}

L'analyse du discours politique peut être réalisée en s'appuyant sur différentes approches disciplinaires (énonciative, pragmatique, herméneutique, stylistique, lexicologique...), chaque approche prenant en considération un aspect spécifique de l'objet discours. Le foisonnement des approches se réclamant de l'analyse du discours rend le choix particulièrement difficile. Dans cette contribution, I'analyse du discours présidentiel s'inspire de l'approche pragmatico-énonciative. Un intérêt particulier est accordé à la dimension performative du discours présidentiel algérien. Le corpus de cette contribution est constitué des discours prononcés par le président algérien Abdelmadjid Teboune entre février 2020 et janvier 2021 et de deux articles de presse parus durant cette même période dans deux journaux (El Watan) et (Le Point).

\footnotetext{
1 Dans le discours institutionnel algérien, c'est l'ex-ministre de la Communication, Hamid Grine, qui a employé le premier ce terme en février 2017 pour désigner les zones non couvertes par la radiodiffusion en Algérie.
} 


\section{Sens littéral vs sens contextuel}

Une définition lexicographique est nécessaire avant d'entreprendre ce travail de recherche.

L'ombre 《ل الظل الفيء/كنف/حضن/حجر/حماية/جناح dans la langue arabe ont essentiellement une connotation culturelle positive. Cette valeur appréciative de l'ombre est justifiée par l'influence de l'environnement sur la langue arabe. Cet idiome s'est développé dans des zones désertiques de la péninsule arabique (Yémen, Arabie saoudite, Émirats arabes unis...) où l'ombre exprime le sens figuré du bien-être, du confort, de la force, de la fierté et de la protection. Dans le texte coranique, qui est une référence pour la langue arabe, l'ombre (الظل) et ses mots

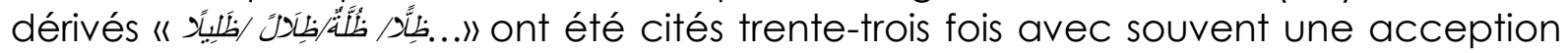
appréciative2. L'ombre évoque dans le Saint Coran la béatitude, le confort, la

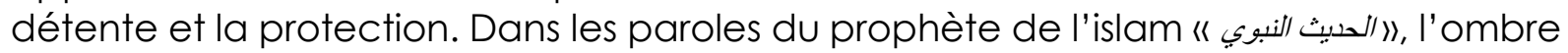
fait référence à la protection divine le jour du dernier jugement. Le hadith des «sept personnes qui seront couvertes par Allah dans Son ombre le jour où il n'y aura pas d'autre ombre que Son ombre " est parmi les paroles du prophète les plus évoquées par les savants musulmans. Dans la poésie arabe post ou antéislamique, l'ombre a souvent une connotation appréciative. Il exprime l'aisance, la quiétude et le confort. Dans la langue française, par contre, l'ombre a souvent une connotation culturelle dépréciative. Pour le dictionnaire Larousse (2020), l'ombre fait référence à une "silhovette sombre, plus ou moins déformée ", une "forme sombre ", une "tache, zone sombre sur un fond plus clair », la "forme indécise de quelqu'un que l'on voit à peine dans une demi-obscurité ", une "très petite quantité de quelque chose, une trace, un soupçon ». Cette dépréciation du lexème " ombre » en français pourrait être expliquée également par l'influence du contexte environnemental. La langue française est née et s'est développée en lle-de-France avant de se répandre dans le monde. Cette région de la France est connue pour son temps morne durant l'automne et l'hiver. Le ciel est désespérément obscur durant plusieurs mois à Paris, ce qui expliquerait en partie l'acception dépréciative de l'ombre. L'expression «zone d'ombre » en français désigne aussi un point obscur et non éclairé.

Surnommée la "ville lumière », Paris a, en effet, longtemps vécu dans l'obscurité, ce qui avait favorisé la criminalité durant la nuit3. Cette brève définition lexicographique révèle que la dimension connotative et culturelle du lexème 《 الظل dans la langue arabe diffère de celle de la langue française. Mais pourquoi le discours politique algérien a-t-il eu recours à cette néologie de sens basée sur un lexème qui a une dimension culturelle appréciative dans la langue arabe et sert à désigner des lieux emblématiques de la misère et de l'exclusion sociale ?

\footnotetext{
2 Dans le verset 16 de la 39 sourate du Coran « Az-Zumar » et le verset 43 de la sourate 56 " Al waqui'a », l'ombre a une connotation dépréciative. Elle est employée comme parasynonyme de « fumée » et de «nuage » pour exprimer le supplice.

3 L'éclairage des rues était défaillant dans ses débuts à Paris. Durant la nuit, les promeneurs redoutaient les mauvaises rencontres avec des agresseurs dans les zones d'ombre. Les zones d'ombre ont ainsi pris le sens en français de l'insécurité et du danger.
} 


\section{Cadre théorique : le figement, une stratégie politique}

Le lexique du discours politique évolve constamment et essentiellement durant les périodes de transitions pour s'adapter aux nouvelles réalités et satisfaire les attentes du public-cible (Gobin, 2011, pp. 169-186). Les nouvelles formules et les néosémies politiques apparaissent suite à un processus de figement linguistique pour soutenir l'idéologie du discours dominant ou au contraire pour la remettre en cause par un processus de défigement (Fiala \& Habert, 1989, pp. 83-99). La notion de figement est, depuis le début des années 1960, au cœur de l'analyse du discours politique en France (Krieg-Planque, 2013, pp. 159-160). L'intérêt pour cette notion a commencé, en réalité, dès les premiers balbutiements de la linguistique moderne. Dans Le Cours de linguistique générale (Saussure, 2016, p. 244), Saussure évoque succinctement l'agglutination, qu'il considère comme un processus qui s'exerce exclusivement dans l'axe syntagmatique. Sechehaye, Bally, Benveniste, Martinet, Rey, Thun, Gaatone, Gross, Mejri et de nombreux linguistes contemporains ont développé des réflexions intéressantes sur le phénomène du figement dans la langue.

Le figement est considéré par ces linguistes comme un processus qui permet de transformer une séquence libre en une unité lexicale figée dans le système de la langue. Dans le dictionnaire de la linguistique et des sciences du langage (Dubois, J. Marcellesi, C., Marcellesi, J. B., Guespin, L., Mevel J.P., Giacomo, M., 2002, p. 202), le figement est défini comme « un processus par lequel un groupe de mots dont les éléments sont libres devient une expression dont les éléments sont indissociables. Le figement se caractérise par la perte du sens propre des éléments constituant le groupe de mots, qui apparaît alors comme une nouvelle unité lexicale, autonome et à sens complet, indépendant de ses composantes \%. Alice Krieg-Planque (2015) note que le figement est "une occasion de resserrement de la créativité verbale, le principe d'imprévisibilité inhérent à l'activité de langage (récursivité, commutabilité, ouverture de la combinatoire...) se trouvant alors restreint ॥. Selon Alice Krieg-Planque (2015, p. 118), le figement, qui est naturellement observable dans les discours politiques, permet de mettre à nu les stratégies de l'autorité en discours.

Le figement linguistique et/ou discursif est une notion féconde pour la linguistique et surtout les sciences du langage. II demeure un champ de réflexion pour l'ensemble des disciplines des sciences du langage : analyse du discours, rhétorique, didactique, sémantique, syntaxe, lexicologie, traductologie, etc. Alice Krieg-Planque (2015, p. 115) souligne dans son article « Construire et déconstruire l'autorité en discours. Le figement discursif et sa subversion » que le figement "participe à la capacité des discours à faire autorité, c'est-à-dire à dissuader la contradiction, à s'imposer sur le mode de l'évidence, à sembler faire consensus ». Cette théoricienne, qui a consacré de nombreux travaux académiques au figement, soutient que cette notion est appropriée voire incontournable pour bien comprendre toutes les manifestations d'autorité en discours. L'usage intentionnel et foncièrement conscient du figement dans le discours politique serait ainsi motivé par des intentions idéologiques. 


\section{4. || Iاطق الظل II, une séquence avec un haut degré de figement}

La constitution d'une séquence figée s'opère par différents procédés linguistiques : le mot composé, le néologisme de sens, l'univerbation, l'agglutination, l'antonomase, la catachrèse, etc. Pour qu'un mot composé ou une locution soient considérés comme une séquence figée, ils doivent nécessairement répondre conjointement à quatre critères:
a. La polylexicalité
b. Le sens global de la séquence séparément de ses composantes : perte du sens propre des constituants

c. L'opacité ou l'ambiguïté sémantique

d. La possibilité du défigement

La polylexicalité est considérée comme une condition nécessaire au figement (Gross, 1996; Mejri, 2004). Le dictionnaire des sciences du langage (Neveu, 2004) précise que la polylexicalité décrit la formation complexe des unités lexicales composées d'au moins deux éléments constitutifs, graphiquement soudés ou non. Les séquences polylexicales ou complexes correspondent, selon (Gross, 1996, p. 7), à «toute unité (catégorie grammaticale ou partie de discours) composée de deux ou de plusieurs mots simples ou mots dérivés préexistants 1 . Une séquence figée perd obligatoirement son sens premier pour renvoyer métonymiquement à un ou des sens contextuel(s). La séquence figée "l مناطق الظل I) a perdu son sens premier, qui désigne des espaces situés dans l'ombre du soleil, pour acquérir un autre sens contextuel renvoyant à des zones exclues du développement, où règne la misère. Le degré de figement d'une séquence est variable d'une formule à une autre. II s'assortit de plusieurs traits distinctifs d'ordre sémantique et syntaxique : la difficulté d'intégration d'une unité nouvelle dans la séquence, l'impossibilité des substitutions parasynonymiques, l'empêchement de la détermination, de l'actualisation, et le blocage des propriétés transformationnelles (pronominalisation, dislocation, relativisation...). Le deuxième critère pour reconnaitre une séquence figée est le sens global de la séquence, indépendamment de ses composantes. Les constituants de cette séquence perdent leur sens premier ou propre pour désigner un sens contextuel. Dans notre séquence figée, "الظل ne désigne ni l'ombre du soleil ni une forme sombre, mais il renvoie, métonymiquement ${ }^{4}$, à un sens figuré : misère, sous-développement, exclusion. Le lexème 《الظل الظ perd son autonomie dans cette séquence figée pour désigner un sens figuré autre que celui prévu par la langue arabe. La séquence étudiée dans cet article est une dénomination et de ce fait elle est nécessairement sémantiquement opaque étant donné que toute formule polylexicale qui évoque une dénomination possède un sens global et non un sens littéral ou compositionnel. Gross (1996, p. 11) précise que dans le cas où le sens d'une suite " n'est pas fonction de celui des éléments constitutifs, (...) nous dirons que nous sommes en présence d'une suite opaque ou sémantiquement figée $\|$.

La possibilité du défigement (Lecler, 2006; Mejri, 2013) est une propriété constitutive de toute expression figée. Le détournement du figement permet non seulement de repérer de manière plus précise les séquences figées et leur degré de figement, mais

\footnotetext{
${ }^{4}$ La séquence figée 《مناطق الظل I est une métonymie (بعدة عن صفة كناية) dans la langue arabe.
} 
il autorise également une meilleure compréhension du processus de conceptualisation de ces expressions toutes faites ou préfabriquées. La séquence figée 《| مناطق الطل ") est défigée sur les réseaux sociaux par des internautes qui lui préfèrent

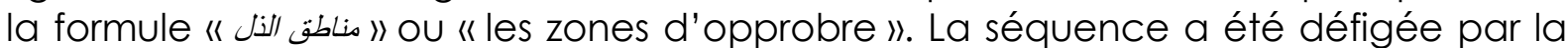

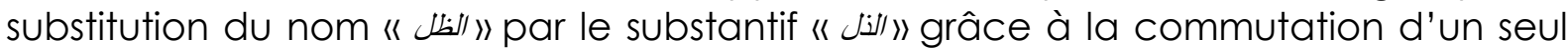
phonème. La lettre (ظ) est substituée par la consonne (ذ). Ces deux phonèmes du système phonologique de la langue arabe sont tous les deux des consonnes fricatives sonores voisées. Le premier phonème (ظ) est une consonne complexe dite emphatique (alvéolaire), alors que le second phonème (j) est une « interdentale ». La séquence figée 《/ هناطق الظل est éligible aux quatre critères exigés par les lexicologues (polylexicalité, sens global, opacité lexicale et possibilité du défigement) et possède de ce fait un haut degré de figement.

\section{5. "L Les zones d'ombre ॥ : un euphémisme lexicalisé ?}

Dans un article du journal «El Watan » du 25 janvier 2021 intitulé «les zones d'ombre, l'autre nom de la pauvreté ॥, la journaliste Nadjia Bouaricha écrit: "Une zone d'ombre est par définition un espace voilé, loin de la lumière et dont les contours sont difficiles à définir. Il se trouve toutefois que la pauvreté en Algérie est tout sauf voilée, elle est flagrante et apparente sauf pour ceux qui ne veulent pas voir $\%$. L'emploi de la séquence « zones d'ombre » serait, selon cette journaliste, un stratagème rhétorique du pouvoir politique pour se "dédouaner de sa mauvaise gestion ». "Qualifier ces régions pauvres de zones d'ombre revient à justifier des décennies de mauvaise gestion. Comme si la pauvreté était une découverte récente en Algérie, on parle de zone d'ombre aujourd'hui en insinuant que le pouvoir central ne connaissait pas leur existence avant et n'a rien vu de la dégradation de la vie dans ces régions jusqu'à ce jour. Une manière de jeter la pierre aux autorités locales et dédouaner des décennies de gestion centralisée sans réel impact sur le développement local. ॥, écrit la journaliste d'El Watan.

Dit autrement, elle qualifie cette séquence figée d'euphémisme destiné à adoucir la réalité amère de ces régions déshéritées. Le plus intéressant dans cet extrait d'article de presse est l'emploi de l'adjectif «voilée » dans l'énoncé « la pauvreté en Algérie est tout sauf voilée ». Selon Jamet :

"L'euphémisme renvoie à une réalité extralinguistique en revêtant une autre forme, un autre signifiant ; c'est ainsi qu'on en parle souvent comme d'un voile jeté sur le signifié, comme pour le camoufler. La métaphore du voile est, on le verra, éclairante, car, contrairement à un tissu épais, le voile laisse entrevoir ce qui se trouve derrière, et le laisse apparaître "semi-cachéu, pourrait-on dire 1 . (2010, p. 33)

Alice Krieg-Planque (2004) confirme que le jugement d'euphémisation est un commentaire ou une évaluation portant sur le rapport de décalage (ratage) entre le mot et la réalité qu'il désigne. Le jugement d'euphémisation serait un "acte savant» qui juge "un terme - et le discours qui le porte - comme le masque fallacieux sous lequel le réel ainsi camouflé est donné à voir " (Krieg-Planque, 2004). La séquence figée 《ل مناطق الظل n'est pas euphémique en elle-même, mais c'est son emploi dans le discours médiatique et le contexte politique qui lui confèrent cette dimension euphémique. Comme le note (Bacry, 1992, p. 105) : "l'euphémisme fait partie de ces procédés (...) qui ne se reconnaissent qu'à l'effet qu'ils produisent ॥. 
L'interprétation de l'euphémisme est intimement liée au contexte de son emploi. Linfoot-Ham, remarque que l'interprétation de l'euphémisme varie selon le contexte socio-politique.

«Interpretation varies according to context, i.e. whether the speaker means the term to be euphemistic, and the hearer interprets it in that light (Warren, 1992). With euphemism being so entwined with context, however, classification of a term as 'euphemistic' becomes difficult. (...) euphemism classification is a grey area, and judgements may differ from person to person. ॥ (Linfoot-Ham, 2005, pp. 228-229)

Le lexicologue (J. Tournier, 1985, p. 261) souligne que l'euphémisme relève de la sociolinguistique, car il s'agit de l'expression d'un fait social lié à la langue. C'est pourquoi Denis Jamet (2010, p. 33) estime que l'euphémisme est d'abord un "processus sociolinguistique »:

« Il est toujours relié à la société dans laquelle il nait, évolve et meurt, et reflète la conception que l'on se fait du réel. Aussi, ce qui peut être un euphémisme pour certains peut apparaitre comme du non-sens ou autre chose pour des personnes extérieures au groupe $॥$.

On serait tenté de conclure que l'expression zones d'ombre serait un euphémisme du politiquement correct ou de la langue de bois. Cette séquence figée ne serait qu'un mot composé atténuant la misère et la situation de détresse des zones exclues du développement et c'est pour cette raison qu'elle est considérée comme polémique et rejetée par ceux qui s'opposent à la communication politique. Le problème avec cette séquence figée est que son intrusion dans le discours politique a eu lieu par le biais de la projection d'un film documentaire dont les images éloquentes révèlent la détresse de la population des zones défavorisées à travers le pays. Les images, plus expressives que les mots, projetées dans ce documentaire de la télévision algérienne remettent partiellement en cause l'idée du doux euphémisme. La séquence figée "الناطق الظل الن est certes un euphémisme par lequel le pouvoir politique essayerait d'adoucir la réalité malheureuse des zones défavorisées, mais l'emploi du substantif «الظل الط serait aussi l'indice d'une autre stratégie rhétorique. L'ombre renvoie dans cette séquence figée à l'idée du désintéressement, de l'omission, de l'oubli, de la dissimulation et de l'abandon. Cette séquence ne remet pas en cause l'ensemble du système politique algérien, mais elle pointe un doigt accusateur sur les responsables locaux (walis, maires, chefs de daïras...). La communication politique tenterait à travers l'emploi de cette séquence de faire croire que le malheur de ces zones est provoqué par l'administration locale. Pourtant, l'Algérie n'est pas un état fédéral et la gestion centralisée des affaires politico-administratives reste, en dépit de nombreuses réformes, l'unique mode de gouvernance. Outre cette centralisation de la gestion des affaires publiques, les prérogatives des élus locaux (APC et APW) sont extrêmement limitées par la réglementation en vigueur. Un autre écueil au développement local est la centralisation de la fiscalité, qui ne profite pas à de nombreuses communes « riches » malgré le fait que celles-ci accueillent des activités industrielles et pétrochimiques ${ }^{5}$. La conséquence de cette centralisation de la fiscalité

\footnotetext{
${ }^{5}$ L'article 31 du code des taxes sur le chiffre d'affaires CTCA stipule que « les redevables centralisant leur chiffre d'affaires au niveau du siège social peuvent déduire dans les mêmes conditions la taxe ayant grevé les biens ou services acquis par ou pour leurs diverses unités, établissements ou exploitations $॥$. L'article 31 bis du même code précise que « nonobstant les dispositions de l'article 32 ci-dessous, les redevables consolidant leurs comptes au niveau de la société mère dans les conditions prévues à l'article
} 
est qu'une grande partie des communes sont déficitaires'. La séquence figée " مناطق الظل justifierait implicitement l'exclusion du développement des zones déshéritées par l'idée de l'omission et de l'oubli. Elle sous-entend que l'Etat a fourni de grands efforts pour le développement du pays, mais que des poches de pauvreté demeurent sur un fond éclairé. L'ombre exprime en français le sens figuré de quantité très petite. II est ainsi parasynonyme de "trace " et de "soupçon ». Cependant, si on se réfère aux chiffres officiels communiqués en juin 2020 par le ministre de l'Intérieur et les Collectivités locales et de l'Aménagement du territoire, on apprend que le pays compte quelques 15.000 zones d'ombre où résident 8,5 millions d'habitants, soit une moyenne de dix zones d'ombre pour chaque commune.

\section{Une séquence figée qui a perdu sa charge euphémique}

Cette séquence figée, qui a commencé comme un euphémisme dans le discours politique algérien, semble perdre sa charge euphémique. Elle est devenue un euphémisme en voie de lexicalisation. Cette expression est ainsi employée dans le langage courant par les citoyens, y compris ceux des zones défavorisées en question. Denis Jamet (2010, p. 34) note que si l'euphémisme est lexicalisé, il perd systématiquement sa charge euphémique pour devenir un lemme consacré par l'usage.

"Les euphémismes évoluent, comme tout signe linguistique, et c'est pour cette raison qu'ils peuvent également être classés selon leur degré de figement (...) Cette question du figement linguistique est primordiale, car si l'euphémisme n'est pas lexicalisé, il y a encore perception par l'énonciateur de la motivation de ce dernier, et par conséquent de la possibilité d'utiliser cette expression aux dépens d'une autre, et l'on se trouve face à un réel choix discursif / énonciatif. Si, au contraire, l'euphémisme est lexicalisé, le choix ne sera pas un réel choix, car le mot euphémique, devenant le terme consacré par l'usage, perd ainsi sa charge euphémique "..

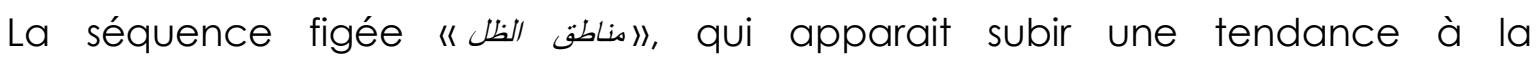
lexicalisation dans la langue quotidienne en Algérie, semble se transformer en un lemme parasynonymique de zones sous-développées et pauvres. Cette séquence n'est plus perçue comme un euphémisme par les locuteurs ordinaires, mais elle est devenue, à force de surmédiatisation, une façon courante de désigner les zones déshéritées.

\section{Diviser par des mots : retour à un contexte de crise politique}

L'apparition de la séquence figée "مناطق الظلس dans le discours politique algérien a eu lieu dans le contexte particulier des deux mois ayant fait suite aux élections présidentielles du 12 décembre 2019. Ce scrutin a enregistré une abstention record de 60\%, soit le taux de participation le plus faible de l'histoire des élections présidentielles en Algérie. Le nombre des votes blancs ou nuls a été également important. Sur les 8.504.346 voix exprimées, le nombre des bulletins nuls a été de 1.243.458, soit un huitième des voix. Les élections présidentielles ont été essentiellement boycottées dans les grandes villes du pays avec des taux de participation

138 bis du CIDTA, peuvent déduire, dans les mêmes conditions, la taxe sur la valeur ajoutée ayant grevé les biens et services acquis par ou pour leurs diverses sociétés membres du groupe ॥

6 II existe, en fait, 1.200 communes déficitaires sur un total de 1.541 en Algérie, selon les statistiques officielles fournies par le DG de la caisse de garantie et de solidarité des collectivités locales. 
extrêmement faibles $(19,68 \%$ à Alger, $0,04 \%$ à Tizi Ouzou, 30,36\% à Constantine, 33,32\% à Oran et $33,90 \%$ à Annaba). Les grandes villes, et en particulier la capitale, sont réputées, à tort ou à raison, être des fiefs de la contestation populaire contre le pouvoir politique. Les médias sociaux, essentiellement Facebook, YouTube, Twitter et Instagram ont eu, en effet, un impact avéré sur le processus de socialisation politique des jeunes algériens dans les grandes villes.

L'émergence de la séquence figée 《ناطق الظلس dans le discours politique n'est pas impromptue, mais elle a été précédée par une polémique intense dans les médias et les réseaux sociaux. La controverse a été enclenchée par un article de Kamel Daoud, un journaliste algérien réputé pour ses positions anti-pouvoir, intitulé « Où en est le rêve algérien? ». Dans cet article, publié le 12 janvier 2020 dans la rubrique "Le Postillon» de l'hebdomadaire français "Le Point", Kamel Daoud explique pourquoi, selon son point de vue, le soulèvement populaire du 22 février 2019 a échové. L'éditorialiste développe l'idée que le pouvoir a réussi à prendre le dessus sur le hirak «élitiste » en " captant les résistances conservatrices de l'Algérie rurale ". Pour Kamel Daoud il y a eu

«D'un côté, des protestataires passionnés, admirables mais piégés dans les grands centres urbains et, de l'autre, une offre de "solution " avec une élection présidentielle qui pare le vide, l'instabilité et donc le cauchemar à la libyenne (...) Épuisés et sans visibilité sur l'avenir, beaucoup, dans l'Algérie profonde, feront le choix pragmatique entre la démocratie et la sécurité 1 .

L'éditorialiste pousse son analyse plus loin en estimant que « le contrôle de la ruralité est la clé du pouvoir en Algérie ॥. Le pouvoir politique aurait ainsi compris l'enjeu de recruter ces "Algériens du pays profond qui votent bien et que les intellectuels délaissent ». L'éditorial de Kamel Daoud est un vrai réquisitoire contre le hirak en Algérie. " ose qualifier Alger de «myope $\|^{7}$ et les espaces urbains en Algérie de "ghetto politique ». " (...) l'Algérie n'est pas la place Maurice-Audin, ni les escaliers de la Grande-Poste ॥, affirme cet éditorialiste. Contrairement aux grandes villes où le taux d'abstention a été important, les zones rurales, considérées comme des territoires conquis par le pouvoir politique, ont enregistré, selon les statistiques officielles, des records de participation dans les élections présidentielles. Les images de bousculades et de chaines humaines interminables devant les bureaux de vote ont été diffusées en boucle sur les chaines de télévision algériennes. Le monde rural est réputée un bastion électoral du régime politique algérien, ce qui expliquerait, en partie, l'engagement de la Présidence au développement de ces zones. Le programme spécial de développement des zones d'ombre 8 , qui a été lancé en 2020 par les

\footnotetext{
7 « Alger souffre en effet d'un nombrilisme qui déteint souvent sur les contestataires. On y croit ce que les journalistes étrangers perpétuent eux aussi, qu'Alger c'est l'Algérie (...) Hors d'Alger, des Algériens ont voté dans le calme et sans scène de violence (...) La ruralité a été perdue par la contestation dès juin, et c'est un constat que les Algérois refusent, souvent avec agressivité. Incapables de sortir de la capitale, d'imaginer un leadership décentralisé et une contestation qui reconnaît au monde rural la paternité de la révolution. Cette myopie trompera lourdement les médias étrangers et les analystes sous influence de militants locaux, ou eux-mêmes correspondants militants non déclarés. Une ceinture de militants-témoins habituels, l'effet de foule sur place, et un accès difficile au pays consacreront cette illusion ॥.

8 Une enveloppe de 110 milliards de dinars a été débloquée, au titre de l'exercice 2021, pour le développement des zones d'ombre.
} 
pouvoirs publics, pourrait être considéré comme une récompense pour ces zones en reconnaissance de leur soutien électoral.

La médiatisation à outrance de cette séquence figée serait également perçue comme une stratégie politique visant à opposer une population urbaine privilégiée, mais contestataire, à une population rurale défavorisée, mais qui vote « bien ». Cette séquence figée ressassée sur tous les médias sème déjà la discorde sur les réseaux sociaux. Les zones rurales sont accusées par les radicaux du mouvement de contestation populaire en Algérie d'être des espaces de soumission et d'opprobre.

\section{Une séquence figée pour reconstruire une identité politique}

Le discours présidentiel algérien est un discours identitaire (Scagnetti, 2002, p. 69). II demeure "très fortement marqué par une recherche d'appartenance, une quête d'identité pour l'individu, le peuple et le pays" (Scagnetti, 2002, p. 69). La question de l'identité dans une conjoncture de révolution populaire semble devenir un défi majeur pour le pouvoir algérien qui, face au boycott massif dans les grandes villes algériennes des élections présidentielles de décembre 2019, s'est tourné vers les zones rurales pour y puiser sa légitimité. Le discours présidentiel algérien s'est appuyé entre 1962 (indépendance du pays) et le 2 avril 2019 (démission d'Abdelaziz Bouteflika) sur la légitimité historique (révolutionnaire) pour exercer le pouvoir (Addi, 2020, p. 104). Les six premiers présidents de la République algérienne étaient tous des anciens combattants (moudjahidines) de l'armée de libération nationale (ALN) qui profitaient de leur statut de « libérateurs » pour en tirer leur légitimité politique. L'actuel président algérien, quant à lui, est un fonctionnaire de carrière qui ne dispose ni de la légitimité historique, ni révolutionnaire, ni démocratique (les élections présidentielles ont été majoritairement boycottées par les électeurs algériens). Le discours présidentiel algérien devait ainsi s'adapter à la nouvelle situation politique pour justifier l'autorité du chef de l'Etat. II semble se contenter de tirer sa légitimité (contestée) de ses bastions électoraux situés dans les zones intérieures et/ou rurales du pays.

Le candidat Abdelmajid Tebboune, soutenu par l'armée algérienne, qui demeure la source de pouvoir en Algérie (Addi, 2020, p. 104), a dès les premiers jours de sa campagne électorale inscrit le développement des zones rurales en tête de ses priorités. Après son investiture, le nouveau président a déclaré que la construction de l'Algérie nouvelle "I ne saurait se concrétiser sans la promotion de ces zones et la prise en charge de leurs populations 1. Lors de sa réunion du 16 février 2020 avec les 48 walis (préfets), il a réitéré sa conviction qu'll on ne saurait parler de nouvelle Algérie sans le développement de ces régions \%. Ces deux déclarations du président algérien sont des actes performatifs qui impliquent l'émergence d'une nouvelle réalité. Le président algérien a justifié, dans son discours du 16 février 2020, son intérêł pour le développement des zones rurales par la nécessité de l'instauration d'une "justice sociale conforme aux exigences de I Etat de droit ॥.

Le discours présidentiel algérien repose ainsi la question de l'identité politique. II devient un lieu de définition de la nouvelle identité algérienne à travers un autre concept, largement médiatisé par l'actuel président, celui d'une « Algérie nouvelle ». Dans le discours présidentiel, l'expression Algérie nouvelle demeure étroitement corrélée aux « zones d'ombre ». Ces zones, oubliées durant plusieurs décennies par le pouvoir algérien, deviennent brusquement dans un contexte de crise politique et sociale l'un des fondements de la nouvelle Algérie. Ce discours identitaire est à 
considérer comme un acte de langage qui transforme le monde. II dispose d'une dimension performative qui a pour objectif de construire une réalité sociale nouvelle (Searle 1995). L'actuel président algérien, originaire des hautes plaines steppiques du sud-ovest algérien et qui a exercé durant de nombreuses années dans les zones intérieures du pays, opte pour une stratégie de légitimation en se référant à son statut afin de rechercher une connivence ou des liens affectifs avec la population des zones intérieures. Le discours présidentiel algérien tente ainsi de reconstruire une nouvelle identité politique en "s'identifiant» à ses partisans (Mayaffre, 2003, pp. 247-264). II s'agit d'une rupture dans la représentation unanimiste (Serres, 2012) du peuple dans le discours présidentiel algérien.

\section{Conclusion}

Le figement lexical est l'un des processus linguistiques qui renvoient au politiquement correct ou à la langue de bois. Un de ses effets est souvent de permettre un euphémisme. La séquence figée 《I مناطق الظل (zones d'ombre) ne peut être considérée comme une traduction littérale, car il s'agit d'une lexicalisation (néologisme de sens) ayant un objectif politique. Le discours présidentiel algérien semble reposer, à travers son emploi corrélativement avec l'expression Algérie nouvelle, la question de l'identité algérienne dans un contexte de crise politique et sociale. Les "zones d'ombre " deviennent ainsi un lieu d'ancrage pour la reconstruction identitaire. Le danger dans la médiatisation à outrance de cette séquence figée est qu'elle divise implicitement les Algériens entre une population urbaine privilégiée, mais contestataire (ingrate), et une population rurale défavorisée, mais qui vote « bien ». Le discours présidentiel algérien, qui a été caractérisé, durant plusieurs décennies, par un discours unanimiste considérant le peuple comme une unité homogène a finalement rompu avec cette représentation simpliste (Serres, 2012). Le président, qui ne peut désormais revendiquer la parole au nom de tout le peuple, en raison du boycott massif des élections présidentielles de 2019, préfère s'identifier à ses bastions électoraux pour justifier sa légitimité.

La séquence figée "مناطق الظل possède un haut degré de figement puisqu'elle répond conjointement à tous les critères syntaxiques et sémantiques nécessaires (polylexicalité, le sens global de la séquence, indépendamment de ses composantes, l'opacité ou l'ambiguïté sémantique et la possibilité du défigement). Cette redondance figée a toutefois perdu sa charge euphémique pour se transformer en un lemme parasynonymique de zones sous développées et pauvres. Elle parait subir un processus de lexicalisation dans l'arabe algérien. Une nouvelle étude pour confirmer le processus de lexicalisation de cette expression dans l'arabe algérien est désormais plus que nécessaire.

\section{Bibliographie}

ADDI, L. (2020). Le système de pouvoir en Algérie, son origine et ses évolutions. Confluences Méditerranée, 115 (4), p. 103.

https://doi.org/10.3917/come.115.0105 [15/05/2021].

BACRY, P. (1992). Les figures de style. Paris : Belin.

BOUARICHA, N. (le 25 janvier 2021). Les zones d'ombre, l'autre nom de la pauvreté, El Watan. https://www.elwatan.com/pages-hebdo/sup-eco/zones-dombrelautre-nom-de-la-pauvrete-25-01-2021 [02/02/2021]. 
DAOUD, K. (le 12 janvier 2020). Où en est le rêve algérien? Comment le soulèvement populaire du 22 février 2019 a-t-il pu échover ?, Le Point.

https://www.lepoint.fr/editos-du-point/sebastien-le-fol/kamel-daoud-ou-en-estle-reve-algerien-12-01-2020-2357340_1913.php [05/02/2021].

Dubois, J. Marcellesi, C., MarCellesi, J. B., GuesPin, L., MeVel J.P. \& Giacomo, M. (2002). Dictionnaire de Linguistique et des Sciences de Langage. Paris : Larousse.

FIALA, P. \& HABERT, B. (1989). La langue de bois en éclat: les défigements dans les titres de presse quotidienne française. Mots, 21 (1), pp. 83-99. https://doi.org/10.3406/mots.1989.1504 [04/01/2021].

FialA, P., HABERT, B., LAFON, P. \& Pineira-TRESmONTANt, C. (1987). Des mots aux syntagmes [Figements et variations dans la Résolution générale du congrès de la CGT de 1978]. Mots, 14 (1), pp. 47-87. https://doi.org/10.3406/mots.1987.1329 [02/01/2021].

GoBIN, C. (2011). Des principales caractéristiques du discours politique contemporain... Semen, pp. 169-186. http://journals.openedition.org/semen/9018 [16/02/2021.

Gross, G. (1996). Les expressions figées en français: noms composés et autres locutions. Paris: Ophrys.

JAMET, D. (2010). Historique et procédés linguistiques de l'euphémisme. In JAMET, D. \& Jobert, M. (ed.), Empreintes de l'euphémisme. Tours et détours. Paris: l'Harmattan.

Krieg-Planque, A. (2004). "Souligner l'euphémisme: opération savante ou acte d'engagement? Analyse du «jugement d'euphémisation» dans le discours politique ». Semen. http://journals.openedition.org/semen/2351 [06/01/2021].

KRIEG-PLANQUE, A. (2013). Le traitement du « figement » par des locuteurs ordinaires : le sentiment linguistique d' "expression toute faite " dans des contextes de critique du discours politique. Pratiques, (159-160), pp. 189-203.

https://doi.org/10.4000/pratiques.2904 [08/01/2021].

KRIEG-PLANQUe, A. (2015). Construire et déconstruire l'autorité en discours. Le figement discursif et sa subversion. Mots, (107), pp. 115-132. https://doi.org/10.4000/mots.21926 [07/01/2021].

LECLER, A. (2006). Le défigement : un nouvel indicateur des marques du figement? Cahiers de praxématique, (46), pp. 43-60. https://doi.org/10.4000/praxematique.596 [29/01/2021]

LINFOOT-HAM, L. (2005). The Linguistics of Euphemism: A Diachronic Study of Euphemism Formation. Journal of Language and Linguistics, 4, pp. 227-263.

https://nanopdf.com/download/the-linguistics-of-euphemism-a-diachronicstudy-of-euphemism_pdf [03/03/2021].

MAYAFFRE, D. (2003). Dire son identité politique. Cahiers de la Méditerranée, (66), pp. 247-264. https://doi.org/10.4000/cdlm.119 [16/05/2021].

MEJRI, S. (2004). Introduction : Polysémie et polylexicalité. Syntaxe et sémantique, 5 (1), p. 13. https://doi.org/10.3917/ss.005.0013 [14/02/2021].

MEJRI, S. (2013). Figement et défigement: problématique théorique. Pratiques, (159-160), pp. 79-97. https://doi.org/10.4000/pratiques.2847 [08/02.2021].

NeVEU, F. (2004). Dictionnaire des sciences du langage. Paris: Armand Colin. SAussure, F. (2016). Cours de lingusitique générale. Paris: Payot et Rivages. 
SCAGNETTI, J. (2002). Analyse du discours politique algérien : I'Algérie et le monde (19621984). Mediterrán tanulmányok, pp. 69-84. http://acta.bibl.u-szeged.hu/37983/ [16/05/2021].

SEARLE, J. (1998). La construction de la réalité sociale. Paris : Gallimard.

SERRES, T. (2012). Variations sur le thème de l'union du peuple dans les discours politiques en Algérie. Dynamiques internationales. https://s3-eu-west1.amazonaws.com/dynamiquesinternationales/DI7/Serres-DI7.pdf [11/05/2021].

TOURNIER, J. (1985). Introduction descriptive à la lexicogénétique de l'anglais contemporain. Paris : Champion-Slatkine.

TOURNIER, M. (1985). Méthodes pour l'étude de la sloganisation. In Langues d'Etat : peuple/pueblo/pays. Spécificités. Sloganisation. Mots, 11, octobre 1985, pp. 155187. 